\title{
O Papel Preditivo da Habilidade Empática sobre o Perdão Interpessoal
}

\author{
Vanessa Dordron de Pinho ${ }^{1}$ \\ Centro Educacional Celso Lisboa, Rio de Janeiro, RJ, Brasil \\ Fundação de Saúde da Prefeitura de Angra dos Reis, Angra dos Reis, RJ, Brasil \\ Eliane Mary de Oliveira Falcone \\ Instituto de Psicologia da Universidade do Estado do Rio de Janeiro, \\ Rio de Janeiro, RJ, Brasil \\ Aline Sardinha \\ Instituto de Psiquiatria da Universidade Federal do Rio de Janeiro, \\ Rio de Janeiro, RJ, Brasil \\ Instituto Nacional de Ciência e Tecnologia Translacional em Medicina, \\ Rio de Janeiro, RJ, Brasil
}

\section{Resumo}

Pesquisas associam benefícios pessoais e interpessoais à empatia, uma habilidade de interação social que abrange aspectos cognitivos, afetivos e comportamentais. A empatia envolve a capacidade para adotar a perspectiva afetiva e cognitiva do outro (tomada de perspectiva), para importar-se genuinamente com o bem-estar alheio (sensibilidade afetiva) e para comportar-se de forma congruente, expressando ao interlocutor que o compreende e se preocupa com o mesmo. Estudos recentes têm relacionado esta habilidade ao perdão interpessoal, o qual caracteriza-se pela mudança cognitiva, afetiva e comportamental em relação a um ofensor específico, de uma polaridade negativa a outra positiva. Neste estudo, objetivou-se avaliar o valor preditivo da empatia sobre o perdão, a partir de medidas de autorrelato multidimensionais. O Inventário de Empatia e o Enright Forgiveness Inventory foram aplicados a uma amostra brasileira, composta por 172 participantes. Análises de regressão indicaram que três fatores empáticos - Tomada de Perspectiva, Sensibilidade Afetiva e Altruísmo - foram preditores do perdão, nas dimensões afetiva e comportamental. Os resultados encontrados apoiam a importância da empatia para a ocorrência do perdão e sugerem que investir nesta habilidade seja um meio promissor de promoção do perdão em intervenções para superação da mágoa.

Palavras-chave: Empatia, perdão, tomada de perspectiva.

\section{The Predictive Role of Empathic Skill on Interpersonal Forgiveness}

\begin{abstract}
Current research point to personal and interpersonal benefits associated to empathy, a social interaction ability that includes cognitive, affective and behavioral aspects. Empathy involves the ability to adopt the other's affective and cognitive perspective (perspective taking), to care genuinely with the welfare of others (affective sensitivity) and to behave in a consistent manner, expressing understanding and
\end{abstract}

Endereço para correspondência: Rua Garibaldi, 133, Bloco A, 301, Tijuca, Rio de Janeiro, RJ, Brasil 20511330. Fone: (21) 98483-3474. E-mail: vanessanessapsi@gmail.com 
concern to the one whom you are talking to. Recent studies have linked this ability to interpersonal forgiveness, which is characterized by cognitive, affective and behavioral change in relation to a specific offender, from a negative polarity to a positive one. This study aimed to evaluate the predictive power of empathy on forgiveness, using self-report multidimensional instruments. The Empathy Inventory and the Enright Forgiveness Inventory were applied to a Brazilian sample composed of 172 participants. Regression analysis indicated three dimensions of empathy - Perspective Taking, Affective Sensitivity and Altruism - as predictors of affective and behavioral aspects of forgiveness. Results support the important role of empathy for the occurrence of forgiveness and they suggest investing in this ability is a promising means of promoting forgiveness in interventions to overcome grief.

Keywords: Empathy, forgiveness, perspective taking.

\section{El Papel Predictivo de la Capacidad Empática sobre el Perdón Interpersonal}

\section{Resumen}

Investigaciones han asociado beneficios personales e interpersonales a empatía, una habilidad de interacción social que incluye aspectos cognitivos, afectivos y conductuales. Empatía implica capacidad de adoptar el punto de vista afectivo y cognitivo de los otros (toma de perspectiva), de preocuparse con el bienestar de los demás (sensibilidad afectiva) y de comportarse de manera consistente, expresando al otro comprensión y que el mismo le importa. Estudios recientes han relacionado esta capacidad al perdón interpersonal, que se caracteriza por el cambio cognitivo, afectivo y de comportamiento en relación con un agresor específico, de una polaridad negativa a otra positiva. Este estudio tuvo como objetivo evaluar el valor predictivo de la empatía sobre el perdón, usando medidas multidimensionales de autorreporte. Se aplicaron el Inventario de Empatía y el Enright Forgiveness Inventory a una muestra brasileña de 172 participantes. Los análisis de regresión indicaron que tres factores de la empatía - Toma de Perspectiva, Sensibilidad Afectiva y Altruismo - fueron predictores del perdón en sus dimensiones afectivas y conductuales. Los resultados apoyan la importancia de la empatía para la ocurrencia del perdón y sugieren que la inversión en esta habilidad es un medio prometedor para promover perdón en intervenciones para superar el dolor.

Palabras clave: Empatía, perdón, toma de perspectiva.

A empatia é um tema de estudo de muitas disciplinas dentro e fora da psicologia (Decety \& Ickes, 2009). Graças a contribuições de diversas áreas de saber, é amplo o conhecimento que se tem atualmente sobre o assunto. Investigações evolucionistas caracterizam a empatia como uma capacidade humana evoluída, compartilhada, em alguns aspectos, com mamíferos e aves, e de valor adaptativo para a sobrevivência das espécies sociais (de Waal, 2010; Eibl-Eibesfeldt, 1970). Além disso, é sabido que manifestações empáticas podem ser observadas em bebês humanos desde seus primeiros dias de vida (Bussab, Pedrosa, \& Carvalho, 2007; Gardner, 2002; Preston \& de Waal, 2002). As experiências de socialização e a maturação cortical ao longo da ontogênse, entretanto, possibilitam manifestações empáticas mais sofisticadas entre os humanos adultos, quando comparados a crianças e a membros de outras espécies animais (Decety \& Jackson, 2004). São objeto de interesse do presente artigo as manifestações empáticas mais sofisticadas. Enquanto habilidade de interação social, a empatia pode ser definida como "a capacidade de compreender, de forma acurada, bem como de compartilhar ou considerar sentimentos, necessidades e perspectivas de alguém, expressando este entendimento de tal maneira que a outra pessoa se sinta compreendida e validada" (Falcone et al., 2008, p. 323). 
A definição acima compreende a empatia como uma habilidade multimensional, com aspectos cognitivos, afetivos e comportamentais e está de acordo com achados neurocientíficos, que indicam que muitos circuitos neurais estão envolvidos neste fenômeno (Baron-Cohen, 2011; Pfeifer \& Dapretto, 2009; Preston \& de Waal, 2002). Dentre as diversas regiões e circuitos neurais associados à experiência empática, pode-se destacar o sistema de neurônios-espelho (relacionado a formas mais primitivas, automáticas e emocionais da empatia) e o córtex pré-frontal (relacionado a formas mais sofisticadas, controladas e cognitivas desta habilidade).

O sistema de neurônios-espelho está distribuído nas áreas corticais frontal e parietal inferior e ventral, é compartilhado com outras espécies animais (Iacoboni, 2009; Lameira, Gawryszewski, \& Pereira, 2006; Rameson \& Lieberman, 2009) e cumpre o papel de dar um entendimento às ações e emoções dos outros sem mediação cognitiva de ordem superior (Oliva, 2012). O córtex pré-frontal é uma região mais recente na história evolutiva, está mais desenvolvido em humanos (Dalgalarrondo, 2011), e possibilita destrezas cognitivas empáticas mais complexas, como flexibilidade para diferenciar a própria perspectiva e a dos demais, autorregulação para modular a angústia desencadeada pela percepção do sofrimento alheio e para modular a raiva decorrente de conflitos interpessoais etc. (Baron-Cohen, 2011; Falcone, 2012; Preston \& de Waal, 2002).

O componente afetivo da empatia pode ser chamado de sensibilidade afetiva (Falcone et al., 2008) e diz respeito à capacidade de se sensibilizar diante da dor do outro e de se interessar genuinamente pelo seu bem-estar. O componente cognitivo pode ser chamado de tomada de perspectiva (Davis, 1983; Decety \& Jackson, 2004; Falcone et al., 2008) e se refere à capacidade de perceber com precisão o ponto de vista e os sentimentos de outra pessoa. Ambos componentes devem estar presentes para caracterizar-se a habilidade empática, senão o que se observa é uma tomada de perspectiva fria ou angústia diante do sofrimento do outro (de Waal, 2010; Falcone, 2012). Além das dimensões cognitiva e afetiva, a empatia, enquanto habilidade interpessoal, também abrange uma dimensão comportamental, expressa por meio de ações verbais e/ou não verbais que denotam uma ação de ajuda, consolo ou demonstração de entendimento (Falcone, 1999).

É importante salientar que, embora o adulto humano tenha destrezas empáticas mais sofisticadas que crianças ou animais de outras espécies, trata-se de um potencial. Ou seja, de acordo com a história desenvolvimental de cada indivíduo, sua capacidade empática pode estar mais ou menos aprimorada. Por exemplo: a tomada de perspectiva pode estar mais acurada em algumas pessoas; a capacidade para modular a angústia pessoal diante do sofrimento do outro pode estar mais funcional em alguns sujeitos; certos indivíduos podem ser mais hábeis em expressar comportamentos empáticos. Ressalta-se, também, que os níveis de empatia podem variar conforme o momento, o contexto, o interlocutor ou devido a outras condições que afetam a experiência.

Muito do interesse pelo tema da empatia se deve ao seu importante papel para a vida em grupo, sendo a mesma considerada um dos alicerces da moralidade humana (Hauser, 2006). Isso ocorre porque a empatia é um dos recursos que permite ao indivíduo equilibrar os interesses pessoais e os interesses do outro.

Estudos indicam que a habilidade empática possui diversos benefícios. Ela é motivadora de comportamentos altruístas (de Waal, 1996, 2008, 2010) e está associada positivamente à inteligência social, ao sucesso profissional, a um maior número de amizades (Ickes, 1997), à satisfação conjugal (Ribeiro, Pinho, \& Falcone, 2011; Sardinha, Falcone, \& Ferreira, 2009), à resiliência (Cecconello \& Koller, 2000) e à melhor adaptação ao estresse (Feshbach, 1997). Por outro lado, a sua ausência está relacionada a prejuízos pessoais e interpessoais (Ickes, 1997) e a diversos transtornos psicológicos, como o autismo e os transtornos de personalidade (Baron-Cohen, 2011).

Mais recentemente, os pesquisadores passaram a investigar o papel da empatia na promoção do perdão interpessoal (e.g. Enright \& Eastin, 1992; McCullough et al., 1998) e os estudos têm encontrado associações positivas entre os 
fenômenos. Quando uma pessoa percebe que foi prejudicada ou ofendida profunda e injustamente por outra, é comum que ela responda à transgressão com emoções (e.g. raiva), pensamentos (e.g. condenação), e comportamentos (e.g. retaliação) negativos (Freedman, Enright, \& Knutson, 2005). A empatia parece ser um dos recursos que facilita a superação dessas respostas negativas, por meio da ampliação do entendimento sobre o ofensor (sua história, suas limitações, seu ponto de vista, o contexto a partir do qual agiu, suas motivações, entre outros) e do desenvolvimento de uma possível compaixão pelo mesmo.

Embora ainda não exista na literatura psicológica um consenso entre os teóricos sobre a definição do perdão (Brown, 2003), a maioria concorda que este envolve a superação de respostas afetivas, cognitivas, comportamentais e motivacionais negativas e o desenvolvimento de outras mais positivas (Worthington, 2005). Por exemplo, para Enright e Rique (2007), o perdão seria a "disposição para abandonar o próprio direito de ressentimento, julgamento negativo e comportamento indiferente em relação a outrem que injustamente nos prejudicou, enquanto encoraja as qualidades desmerecidas de compaixão, generosidade e mesmo de amor em direção a essa pessoa" (p. 4).

Existem evidências de que a empatia é um fator facilitador do perdão interpessoal. Modelos de tratamento propostos para a promoção do perdão enfatizam o desenvolvimento da empatia pelo ofensor e os resultados têm sido promissores na superação da mágoa e emergência do perdão (Pinho \& Falcone, 2015). Além dos estudos clínicos, pesquisas empíricas, por meio da aplicação de instrumentos de autorrelato de empatia e de perdão, também têm encontrado associações positivas entre os fenômenos.

McCullough et al. (1998), por exemplo, validaram uma medida de perdão, o Transgression-Related Interpersonal Motivations Inventory (TRIM), e a correlacionaram a vários instrumentos, dentre os quais a uma medida de empatia afetiva, a Batson Empathy Adjectives (BEA; Coke, Batson, \& McDavis, 1978), em uma amostra de 187 estudantes universitários. Eles encontraram que aumentos em empatia estão relacionados à redução em motivações evitativas e vingativas. Em outro estudo, Hodgson e Wertheim (2007) usaram uma medida multidimensional de empatia (IRI; Davis, 1983) e unidimensional de perdão interpessoal (TFS; Berry, Worthington, O'Connor, Parrott, \& Wade, 2005). Dentre os resultados, a tomada de perspectiva foi o único preditor significativo do perdão. A preocupação empática (fator afetivo da empatia) não teve correlações significativas com o perdão. Outros estudos, com diferentes instrumentos de mensuração, também encontraram relações positivas entre componentes cognitivos e/ou afetivos da empatia e o perdão interpessoal (e.g. Berry, Worthington, Parrott, O'Connor, \& Wade, 2001; Berry et al., 2005).

O presente trabalho teve como objetivo avaliar a influência da habilidade empática sobre o perdão interpessoal, a partir de uma metodologia diferenciada da de pesquisas anteriores. O estudo se deu por meio da aplicação de medidas de autorrelato que contemplam ambos, a empatia e o perdão, como construtos multidimensionais. Pela primeira vez, o Inventário de Empatia (IE) foi utilizado em associação com uma medida de perdão. Ainda, pesquisas sobre o perdão são incipientes na literatura brasileira e é relevante investigar se os fenômenos estão relacionados positivamente conforme ocorre em estudos de âmbito internacional.

Conjecturou-se que a tomada de perspectiva (TP) seria o principal componente empático a ter valor preditivo sobre o perdão interpessoal. Essa hipótese decorre do modelo teórico proposto por Falcone (2012) de que existem duas vias da empatia, uma automática e outra controlada, e que destaca o papel da TP em ambas as vias. Na via automática, o elemento afetivo seria desencadeado primeiramente e a TP cumpriria o papel de modulação da experiência empática emocional. $\mathrm{Na}$ via controlada, através de um esforço consciente para adotar a perspectiva do outro, a TP levaria à experiência afetiva de compaixão em relação ao interlocutor. A ocorrência do perdão seria contemplada na segunda via. Através do esforço da mente para compreender o ofensor, a compaixão e o perdão seriam respostas subsequentes. Com relação aos outros fatores da me- 
dida de empatia empregada, não foram conjecturadas hipóteses prévias acerca do valor preditivo sobre o perdão interpessoal.

\section{Método}

\section{Participantes}

A amostra de conveniência foi constituída por 172 indivíduos adultos, 117 (68\%) mulheres e $55(32 \%)$ homens. A idade variou de dezoito a 76 anos $(M=35,5 ; D P=12,9)$. A maioria dos participantes era casada $(n=83)$ ou solteira $(n=77)$. Nove pessoas eram divorciadas e duas, viúvas. A média de escolaridade foi de treze anos de estudos $(D P=2,4)$.

\section{Instrumentos}

Ficha do participante: foi usada para a coleta dos seguintes dados sociodemográficos: gênero, idade, escolaridade e estado civil.

Inventário de Empatia (IE; Falcone et al., 2008): é uma medida de autorrelato, de quarenta itens, que avalia quatro fatores empáticos e que deve ser respondida com base em uma escala de cinco pontos, que varia de (1) - "nunca" a (5) "sempre". Os quatro fatores do IE são: Tomada de Perspectiva (TP), Flexibilidade Interpessoal (FI), Altruísmo (Al) e Sensibilidade Afetiva (SA). A TP refere-se à capacidade de entender a perspectiva e os sentimentos da outra pessoa. A FI indica a capacidade para tolerar comportamentos, atitudes e pensamentos dos outros que são muito diferentes dos próprios ou provocadores de frustração. $\mathrm{O} \mathrm{Al}$ reflete a capacidade para sacrificar os próprios interesses momentaneamente com a finalidade de beneficiar ou ajudar alguém. A SA denota sentimentos de compaixão e de interesse pelo estado emocional do outro.

Os índices de confiabilidade das escalas do IE no estudo de desenvolvimento e validação da medida foram: $\alpha=0,85$ para o fator TP; $\alpha=0,78$ para o fator FI; $\alpha=0,75$ para o fator $\mathrm{Al} ; \alpha=0,72$ para o fator SA. No estudo atual, a consistência interna foi: $\alpha=0,82$ para o fator TP; $\alpha=0,80$ para o fator FI; $\alpha=0,75$ para o fator $\mathrm{Al} ; \alpha=0,80$ para o fator SA. O IE não apresenta um escore global de empatia, apenas se calculam os escores dos fatores.
O estudo de validade convergente do IE foi feito recentemente (Falcone et al., 2013), correlacionando-o à Escala Multidimensional de Reatividade Interpessoal (EMRI) - adaptada da Interpersonal Reactivity Scale (IRI; Davis, 1983) para a população brasileira. Os fatores cognitivos do IE (TP e FI) se correlacionaram positiva e significativamente com o fator cognitivo da EMRI (Tomada de Perspectiva). O fator SA do IE correlacionou-se significativa, porém fracamente, com o fator Consideração Empática (CE) do EMRI. Isso parece ser devido ao fato de que, embora ambos os fatores reflitam consideração e preocupação com os outros, a subescala SA parece indicar uma preocupação mais generalizada com as outras pessoas, enquanto a subescala CE parece representar um componente mais relacionado ao contágio emocional. Esses resultados contribuíram para aprimorar as qualidades psicométricas do IE.

The Enright Forgiveness Inventory, na versão em português, validada para amostras brasileiras, com o nome Escala de Atitudes (EFI; Enright \& Rique, 2007): é um instrumento de autorrelato que avalia o perdão como um fenômeno multidimensional. Há uma escala global que se subdivide em três dimensões, as quais avaliam os aspectos afetivo, cognitivo e comportamental do perdão interpessoal. O EFI global abrange 60 itens objetivos, e cada fator é composto por 20 itens, os quais devem ser respondidos usando uma escala de concordância de seis pontos (1 - Discordo Fortemente, 6 - Concordo Fortemente). O perdão é indicado pelo aumento de afetos, julgamentos e comportamentos positivos em relação a um ofensor e ofensa específicos. O EFI está validado para diversos países, incluindo o Brasil.

No estudo de Subkoviak et al. (1995), de desenvolvimento e validação da EFI, a confiabilidade da escala global entre os sessenta itens foi: $\alpha=0,98$. As subescalas afetiva, cognitiva e comportamental apresentaram todas o mesmo coeficiente de Cronbach: $\alpha=0,97$. Na presente amostra, os índices de confiabilidade entre os itens foram: $\alpha=0,96$ para as subescalas cognitiva e comportamental; $\alpha=0,95$ para a subescala afetiva. O índice de confiabilidade da EFI global foi: $\alpha=0,98$. 


\section{Procedimentos}

$\mathrm{O}$ projeto de pesquisa, de protocolo 022.3.2010, foi submetido e aprovado pelo Comitê de Ética em Pesquisa da Universidade do Estado do Rio de Janeiro (COEP-UERJ). Em seguida, os participantes foram contatados com base na rede social dos pesquisadores. Eles receberam informações sobre o estudo e aqueles que aceitaram colaborar com a investigação assinaram o Termo de Consentimento Livre e Esclarecido e responderam às medidas de autorrelato e à ficha do participante. A aplicação dos instrumentos foi individual, sendo que a maioria optou por respondê-los em casa e devolvê-los em data previamente combinada.

\section{Análise dos Dados}

Foram realizados os seguintes procedimentos estatísticos: análise de variância (para verificar se participantes masculinos e femininos e de diferentes estados civis se diferenciavam nas escalas de perdão), análise de correlação de Pearson (para investigar relações entre idade, escolaridade e as medidas de autorrelato empregadas) e análise de regressão múltipla (para avaliar a predição da empatia -IE - sobre o perdão - EFI)

\section{Resultados}

\section{Análises de Variância}

Os resultados indicaram que não houve diferenças significativas estatisticamente entre homens e mulheres nas escalas de perdão: $t(170)=$ $0,48, p>0,05$ (escala EFI global); $t(170)=0,44$, $p>0,05$ (escala EFI cognitiva); $t(170)=0,66$, $p>0,05$ (escala EFI comportamental); e $t$ (170) $=0,27, p>0,05$ (escala EFI afetiva). As diferenças entre os grupos de estado civil também não foram significativas quanto ao perdão interpessoal: $F(3,178)=2,50, p>0,05$ (EFI global); $F(3,178)=2,20, p>0,05$ (EFI cognitiva); $F$ $(3,178)=2,40, p>0,05$ (EFI comportamental); $F(3,178)=2,60, p>0,05$ (EFI afetiva). Sendo assim, as variáveis sexo e estado civil não foram relevantes para a análise de regressão.

\section{Relações Bivariadas}

A Tabela 1 apresenta o índice das correlações de Pearson e a significância estatística entre as variáveis estudadas. Como esperado, a TP foi o fator empático que mais apresentou correlações positivas significativas com o perdão interpessoal: $r=0,16, p<0,05$ (EFI global); $r=$ $0,17, p<0,05$ (EFI afetiva); e $r=0,18, p<0,01$ (EFI comportamental). Relações significativas estatisticamente foram observadas também entre outras dimensões da empatia e do perdão: entre SA e perdão global $(r=0,13, p<0,05)$; entre SA e perdão comportamental $(r=0,20, p$ $<0,01)$; entre Al e perdão global $(r=0,15, p<$ $0,05)$; e entre $\mathrm{Al}$ e perdão comportamental $(r=$ $0,18, p<0,05)$.

Um dos fatores do IE, a FI, não apresentou correlações significativas estatisticamente com as escalas do EFI: $r=0,03, p>0,05$ (EFI global); $r=0,01, p>0,05$ (EFI cognitiva e afetiva); $r=0,06, p>0,05$ (EFI comportamental). Da mesma forma, a subescala cognitiva do EFI não se correlacionou significativamente com o IE: $r$ $=0,11, p>0,05$ (com TP); $r=0,08, p>0,05$ (com SA); $r=0,01, p>0,05$ (com FI); $r=0,13$ $p>0,05$ (com Al).

A idade e a escolaridade não se relacionaram de modo significativo estatisticamente ao perdão e à empatia: idade e TP $(r=-0,01, p>0,05)$; idade e SA $(r=0,05, p>0,05)$; idade e FI $(r=0,02$, $p>0,05)$; idade e $\mathrm{Al}(r=-0,14, p>0,05)$; idade e EFI global $(r=-0,08, p>0,05)$; idade e EFI cognitiva $(r=-0,12, p>0,05)$; idade e EFI afetiva $(r$ $=-0,06, p>0,05)$; idade e EFI comportamental $(r=-0,05, p>0,05)$; escolaridade e TP $(r=0,02$, $p>0,05)$; escolaridade e SA $(r=0,01, p>0,05)$; escolaridade e FI $(r=0,03, p>0,05)$; escolaridade e $\mathrm{Al}(r=0,08, p>0,05)$; escolaridade e EFI global $(r=-0,05, p>0,05)$; escolaridade e EFI cognitiva $(r=-0,05, p>0,05)$; escolaridade e EFI afetiva $(r=-0,04, p>0,05)$; escolaridade e EFI comportamental $(r=-0,06, p>0,05)$.

Assim, a idade e a escolaridade não foram inseridas no cálculo de regressão. A Tabela 1 também indica que os índices de consistência interna das medidas empregadas foram satisfatórios $(\alpha>0,8)$. Apenas o Al teve o índice um pouco abaixo $(\alpha=0,75)$. 
Tabela 1

Média (DP), Alpha de Cronbach e Correlação entre as Subescalas do Inventário de Empatia (IE), Dimensões da Escala de Atitudes (EFI), Idade e Escolaridade

\begin{tabular}{|c|c|c|c|c|c|c|c|c|c|c|c|c|c|}
\hline & $M$ & $D P$ & $\alpha$ & 1 & 2 & 3 & 4 & 5 & 6 & 7 & 8 & 9 & 10 \\
\hline 1.TP & 42,67 & 7,11 & 0,82 & & $0,50 * *$ & 0,10 & $0,18^{*}$ & $0,16^{*}$ & 0,11 & $0,17^{*}$ & $0,18 * *$ & $-0,01$ & 0,02 \\
\hline 2.SA & 37,30 & 5,10 & 0,80 & & & 0,07 & $0,35 * *$ & $0,13 *$ & 0,08 & 0,08 & $0,20 * *$ & 0,05 & 0,01 \\
\hline 3.FI & 29,41 & 6,77 & 0,80 & & & & $0,42 * *$ & 0,03 & 0,01 & 0,01 & 0,06 & 0,02 & 0,03 \\
\hline 4.Al & 30,80 & 6,12 & 0,75 & & & & & $0,15^{*}$ & 0,13 & 0,11 & $0,18^{*}$ & $-0,14$ & 0,08 \\
\hline 5.EFI Global & 268,40 & 64,19 & 0,98 & & & & & & $0,93 * *$ & $0,97 * *$ & $0,96 * *$ & $-0,08$ & $-0,05$ \\
\hline 6.EFI Cognitiva & 95,70 & 20,90 & 0,96 & & & & & & & $0,84 * *$ & $0,82 * *$ & $-0,12$ & $-0,05$ \\
\hline 7.EFI Afetiva & 85,22 & 23,31 & 0,95 & & & & & & & & $0,90 * *$ & $-0,06$ & $-0,04$ \\
\hline $\begin{array}{l}\text { 8.EFI } \\
\text { Comportamental }\end{array}$ & 87,51 & 23,40 & 0,96 & & & & & & & & & $-0,05$ & $-0,06$ \\
\hline 9.Idade & 35,50 & 12,90 & & & & & & & & & & & $0,15^{*}$ \\
\hline 10.Escolaridade & 13,00 & 2,40 & & & & & & & & & & & \\
\hline
\end{tabular}

Nota. TP (Tomada de Perspectiva), SA (Sensibilidade Afetiva), FI (Flexibilidade Interpessoal), Al (Altruísmo) e EFI (Escala de Atitudes).

${ }^{*} p<0,05$; bicaudado. ${ }^{*} p<0,01$; bicaudado.

\section{Relações Multivariadas}

No artigo original de validação do EFI, Subkoviak et al. (1995) encontraram uma alta correlação entre as subescalas afetiva, cognitiva e comportamental do EFI, justificando o uso de um escore global do perdão. Contudo, os autores indicam que também é importante estudar as subescalas em separado, o que pode trazer contribuições para a melhor compreensão do conceito e processo do perdão. Por essa razão, uma série de análises de regressão múltiplas foram realizadas, de modo que, em cada análise, uma das medidas de perdão era a variável dependente (VD), com exceção da subescala cognitiva, que não teve correlações significativas com os fatores do IE. As variáveis preditoras inseridas nas análises foram aquelas que se relacionaram significativamente com a VD em questão. Os resultados podem ser observados na Tabela 2 .

A TP, a SA e o Al predisseram, em conjunto, $4 \%$ da variabilidade na escala global do EFI. A TP foi a variável que mais contribuiu para a predição do perdão global. Contudo, as variáveis previsoras, individualmente, não tiveram uma contribuição significativa para o modelo: TP $(\beta$ $=0,14 ; p>0,05)$; SA $(\beta=0,02 ; p>0,05) ; \mathrm{Al}(\beta$ $=0,12 ; p>0,05)$.

A TP $(\beta=0,17 ; p<0,05)$ foi a única variável do IE que teve correlação significativa com o perdão afetivo, e foi responsável por $3 \%$ da variabilidade nesta medida. $\mathrm{O}$ perdão comportamental teve $6 \%$ de sua variabilidade explicada pela TP, SA e Al, conjuntamente. O Al foi o fator empático mais importante na predição do perdão comportamental, porém as variáveis, individualmente, não tiveram contribuição significativa para o modelo: $\mathrm{TP}(\beta=0,11 ; p>0,05)$; $\mathrm{SA}(\beta=0,11 ; p>0,05) ; \mathrm{Al}(\beta=0,12 ; p>0,05)$.

\section{Discussão}

O objetivo do presente trabalho foi investigar as relações entre o Inventário de Empatia (IE) e o Enright Forgiveness Inventory (EFI) e avaliar o valor preditivo da empatia sobre o perdão interpessoal. Tanto o IE quanto o EFI são medidas multidimensionais, o que permitiu identificar com mais precisão como os aspectos 
Tabela 2

Variáveis Preditoras do Perdão Interpessoal (EFI)

\begin{tabular}{lccccc}
\hline \multirow{2}{*}{ Preditores } & \multicolumn{2}{c}{ EFI Global } & \multicolumn{2}{c}{ EFI Afetiva } & \multicolumn{2}{c}{ EFI Comportamental } \\
\cline { 2 - 6 } & $\beta$ & $R^{2}$ & $\beta$ & $R^{2}$ & $\beta$ \\
Enter & & $0,04^{*}$ & $0,03^{*}$ & $0,06^{*}$ \\
& & $F(3,169)=3,60, p<0,05$ & & $F(1,170)=5,00, p<0,05$ & $F(3,168)=4,00, p<0,05$ \\
TP & 0,14 & $0,17^{*}$ & 0,11 \\
SA & 0,02 & --- & 0,11 \\
Al & 0,12 & --- & 0,12 \\
\hline
\end{tabular}

Nota. TP (Tomada de Perspectiva), SA (Sensibilidade Afetiva), Al (Altruísmo) e EFI (Escala de Atitudes). ${ }^{*} p<0,05$; unicaudado.

cognitivos, emocionais e comportamentais desses construtos estão relacionados.

Os resultados apontaram que o perdão foi predito por três fatores do IE, Tomada de Perspectiva (TP), Sensibilidade Afetiva (SA) e Altruísmo (Al), o que indica que a empatia tem influência sobre o perdão interpessoal, corroborando a literatura sobre esses temas. Contudo, as dimensões empáticas se relacionaram de modos distintos com as escalas de perdão, o que aponta para a importância da sugestão dada por Subkoviak et al. (1995), acerca do uso das subescalas do EFI em separado.

A TP, a SA e o Al tiveram valor preditivo sobre o comportamento de perdoar, ao passo que apenas a TP predisse a dimensão afetiva do perdão. Esse resultado indica que a capacidade de perceber a perspectiva cognitiva e os sentimentos do outro, a preocupação com o bem-estar do interlocutor e a disposição para abrir mão dos próprios interesses em prol de outrem afetam o desenvolvimento de comportamentos positivos em relação ao ofensor (por exemplo, se aproximar e ajudar). Ainda, a habilidade para adotar a perspectiva do outro contribui para a experiência de afetos mais positivos em relação ao ofensor (por exemplo, sentir carinho).

Conforme fora conjecturado, a TP foi a dimensão da empatia que se mostrou mais relevante para o perdão interpessoal (dado que afetou o perdão nos níveis afetivo e comportamental, ao passo que as demais dimensões tiveram predição apenas sobre a dimensão comportamental). Uma vez que o processo de perdoar alguém por quem se sente raiva, mágoa e outros afetos negativos é árduo e difícil (Freedman et al., 2005), é provável que, para conseguir perdoar o ofensor, muito esforço da mente seja exigido da vítima, que necessita mobilizar diversos processos cognitivos complexos, envolvidos na adoção da perspectiva do outro, para chegar ao perdão.

O perdão demanda a superação de emoções negativas desencadeadas pela ofensa interpessoal sofrida, bem como a expressão funcional de comportamentos em relação ao ofensor. Era esperado, portanto, que a TP, um processo cognitivo superior, relacionado à autorregulação, à flexibilidade cognitiva, às funções de controle executivo e a outros fenômenos mentais complexos (Decety \& Jackson, 2004), influenciasse o perdão. A TP tem sido de grande interesse para os profissionais que buscam ajudar seus clientes a perdoar (e.g. Enright \& Eastin, 1992), sendo um recurso que auxilia a dar maior inteligibilidade às ações de um ofensor. Além disso, estudos (e.g. Ribeiro et al., 2011) têm destacado a relevância da TP na modulação de emoções raivosas e na expressão manifesta funcional dessas emoções.

Esse processo empático permite o conhecimento do mundo subjetivo do outro, suas intenções, fraquezas e limitações, e o entendimento do que motiva suas ações, levando a uma compreensão mais ampla do ponto de vista do outro e à regulação das próprias emoções e comportamentos, o que possibilita a concessão do perdão. Conforme o modelo proposto por Falcone (2012), sobre as duas vias da empatia, este estudo dá respaldo à compreensão de que em casos de conflito e mágoa interpessoais, a TP precisa 
ser mobilizada, através de um esforço cognitivo, para dar sentido às ações do ofensor. Com isso, emoções mais positivas em direção ao agressor podem vir a ocorrer. A via controlada da empatia, portanto, seria a via requerida para alcançar o perdão interpessoal.

Assim, a TP é relevante para que um indivíduo consiga transformar o que sente e o modo como se comporta em relação aos seus ofensores. Porém, esperava-se que adotar a perspectiva do outro fosse contribuir para mudar também as cognições sobre um ofensor, o que não pôde ser verificado com esta pesquisa, uma vez que a subescala cognitiva do EFI não se relacionou à TP, e a nenhuma dimensão do IE.

Esse resultado é surpreendente e não conclusivo. Essa é a primeira investigação que associa o IE e o EFI, de modo que estudos adicionais seriam necessários. No entanto, o resultado encontrado também leva a um questionamento sobre o modo como o perdão cognitivo é avaliado pelo EFI. A sua subescala cognitiva reflete uma mudança de opinião em relação ao ofensor. Mas acredita-se que, ao contrário do proposto por esse instrumento, ao perdoar, passa-se a ter uma visão mais abrangente e complexa sobre o ofensor, o que não significa mudar a opinião que se tem sobre ele. Sendo este o caso, as subescalas afetiva e comportamental do EFI parecem refletir melhor a natureza do perdão que a subescala cognitiva, o que permite a conclusão de que a TP é preditora do perdão, porém não prediz a mudança de opinião que se tem sobre o ofensor. Assim, entender o ofensor pode conduzir à libertação da raiva, ao desenvolvimento de afetos positivos, a comportamentos de ajuda e de consideração, porém a opinião sobre o transgressor pode continuar a mesma.

Outro resultado encontrado foi a falta de relações entre a FI e o perdão. A flexibilidade é uma capacidade cognitiva que contribui para a habilidade de tomar a perspectiva do outro (Decety \& Jackson, 2004). Sendo assim, alguma correlação deveria ter sido encontrada entre esse fator do IE e o perdão, mas isso não foi demonstrado no estudo atual. Como o IE é uma medida de empatia relativamente nova e em processo de validação, acredita-se que a escala de FI precise ainda ser melhor analisada, a fim de verificar se de fato está medindo o construto que pretende avaliar. Enquanto isso, estudos adicionais relacionando flexibilidade e perdão merecem maior atenção empírica.

O altruísmo é uma capacidade predisposta pela empatia (de Waal, 1996, 2008, 2010) e contemplada pelo IE. Embora não tenham sido encontrados, durante a revisão da literatura, estudos empíricos que pesquisassem o papel do altruísmo sobre o perdão, foi possível realizar essa investigação no presente estudo. Como resultado, verificou-se que a disposição para abrir mão dos próprios interesses em prol do outro foi importante para o desenvolvimento de comportamentos positivos em relação ao ofensor. Esse resultado é compreensível quando se raciocina que o altruísmo se manifesta por meio de comportamentos direcionados ao outro. Ajudar, tratar delicadamente e fazer um favor são exemplos de itens da subescala comportamental do EFI que denotam uma ação altruísta para com um ofensor.

Dentre os resultados desta pesquisa, chama atenção ainda o fato de a SA não ter se relacionado à subescala afetiva do EFI. Esses resultados são semelhantes aos de Hodgson e Wertheim (2007), que encontraram ser a TP o preditor mais importante do perdão.

É preciso destacar, contudo, que o IE e o EFI são instrumentos com níveis de especificidade diferentes. Enquanto o IE mede a tendência de um indivíduo para ser empático de um modo geral, o EFI não mede a tendência geral de um indivíduo para perdoar. O EFI avalia o perdão em relação a uma ofensa e a um ofensor específicos. Raciocina-se, deste modo, que a disposição para ter compaixão pelos outros não seja a mesma coisa que ter, de fato, compaixão por um ofensor. Se ao invés de avaliar a disposição empática tivesse sido avaliada a empatia em relação a um ofensor, talvez a SA predissesse afetos positivos em relação ao transgressor.

Tendo em vista os resultados apresentados neste artigo, considera-se relevante que investigações futuras se preocupem em estudar o valor preditivo da empatia sobre o perdão a partir de medidas com um mesmo nível de especificidade. 
É importante estudar a associação entre a empatia a um ofensor específico e o perdão ao mesmo ofensor. Cogita-se que o nível de empatia em relação ao transgressor afetará o nível de perdão em relação ao mesmo.

$O$ fato de o IE e o EFI serem instrumentos com níveis de especificidade diferentes também pode explicar as correlações fracas entre as medidas e o baixo percentual com que os fatores do IE explicaram a variância nas subescalas do EFI. Dada essa possibilidade, sugere-se o desenvolvimento de uma escala que avalie a empatia em relação a ofensores, assim como devem ser criadas, ou validadas para a população brasileira, medidas de perdão disposicional. No momento da realização desta pesquisa, o EFI era a única escala de perdão validada para a população brasileira.

A presente pesquisa apresenta limitações, sendo a principal o fato de o IE e o EFI serem instrumentos com níveis de especificidade diferentes, de modo que isso pode ter impedido que algumas expectativas teóricas fossem confirmadas. Também são necessárias formas de avaliar a empatia e o perdão a partir do ponto de vista de informantes, e não apenas do próprio indivíduo que se autopercebe. Ainda, foi realizado um estudo de natureza correlacional, com medidas de autoinforme. Os resultados são animadores, mas estudos longitudinais e experimentais precisam ser feitos para verificar a relação causal entre a empatia e o perdão.

Ainda assim, os resultados confirmaram a influência da empatia sobre o perdão, conforme a literatura internacional aponta. Como pontos fortes desse estudo, foram usadas medidas multidimensionais tanto da empatia quanto do perdão e a amostra de adultos foi diversificada, não apenas composta por estudantes universitários, como costuma ocorrer nos estudos empíricos sobre o perdão. Esse trabalho também teve o mérito de contribuir para pesquisas sobre o tema no contexto brasileiro.

Por fim, os resultados constatados trazem implicações práticas importantes. Eles sustentam a importância de se investir no desenvolvimento da habilidade empática como um meio de promover o perdão interpessoal. Enfatiza-se, aqui, a importância de aprimorar a capacidade de tomar a perspectiva do ofensor como um meio para a libertação da dor da mágoa e para alcançar o perdão.

\section{Referências}

Baron-Cohen, S. (2011). The science of evil: On empathy and the origins of cruelty. New York: Basic Books.

Berry, J. W., Worthington, E. L., Jr., Parrott, L., III, O'Connor, L. E., \& Wade, N. G. (2001). Dispositional forgivingness: Development and construct validity of the Transgression Narrative Test of Forgivingness (TNTF). Personality and Social Psychology Bulletin, 27(10), 1277-1290. doi: $10.1177 / 01461672012710004$

Berry, J. W., Worthington, E. L., Jr., O'Connor, L. E., Parrott, L., III, \& Wade, N. G. (2005). Forgivingness, vengeful rumination, and affective traits. Journal of Personality, 73(1), 183-226. doi:10.1111/j.1467-6494.2004.00308.x

Brown, R. P. (2003). Measuring individual differences in the tendency to forgive: Construct validity and links with depression. Personality and Social Psychology Bulletin, 29(6), 759-771. doi:10.1177/0146167203029006008

Bussab, V. S. R., Pedrosa, M. I., \& Carvalho, A. M. A. (2007). Encontros com o outro: Empatia e intersubjetividade no primeiro ano de vida. Psicologia USP, 18(2), 99-133. doi:10.1590/S010365642007000200007

Cecconello, A. M., \& Koller, S. H. (2000). Competência social e empatia: Um estudo sobre resiliência com crianças em situação de pobreza. Estudos de Psicologia (Natal), 5(1), 71-93. doi:10.1590/ S1413-294X2000000100005

Coke, J. S., Batson, C. D., \& McDavis, K. (1978). Empathic mediation of helping: A two-stage modelo. Journal of Personality and Social Psychology, 36(7), 752-766.

Dalgalarrondo, P. (2011). Evolução do cérebro: Sistema nervoso, psicologia e psicopatologia sob a perspectiva evolucionista. Porto Alegre, RS: Artmed.

Davis, M. H. (1983). Measuring individual differences in empathy: Evidence for a multidimensional approach. Journal of Personality and Social Psychology, 44(1), 113-126. doi:10.1037/00223514.44.1.113 
De Waal, F. (1996). Good natured: The origins of right and wrong in humans and other animals. London: Harvard University Press.

De Waal, F. (2008). Putting the altruism back into altruism: The evolution of empathy. The Annual Review of Psychology, 59, 279-300. doi:10.1146/ annurev.psych.59.103006.093625

De Waal, F. (2010). A era da empatia: Lições da natureza para uma sociedade mais gentil. São Paulo, SP: Companhia das Letras.

Decety, J., \& Ickes, W. (2009). Introduction. In J. Decety \& W. Ickes (Eds.), The Social Neuroscience of Empathy (pp. VI-IX). Cambridge, MA: A Bradford Book.

Decety, J., \& Jackson, P. L. (2004). The functional architecture of human empathy. Behavioral and Cognitive Neuroscience Reviews, 3, 71-100. doi: $10.1177 / 1534582304267187$

Eibl-Eibesfeldt, I. (1970). Vantagens da sociabilidade. In Amor e ódio. História natural dos padrões elementares do comportamento (pp. 81-86). Lisboa: Livraria Bertrand.

Enright, R. D., \& Eastin, D. L. (1992). Interpersonal forgiveness within the helping professions: An attempt to resolve differences of opinion. Counseling \& Values, 36(2), 84-103. doi:10.1002/ j.2161-007X.1991.tb00966.x

Enright, R. D., \& Rique, J. (2007). The Enright Forgiveness Inventory (EFI). User's Manual. Madison, WI: Mind Garden.

Falcone, E. M. O. (1999). A avaliação de um programa de treinamento da empatia com universitários. Revista Brasileira de Terapia Comportamental e Cognitiva, 1(1), 23-32. Recuperado em http://www.usp.br/rbtcc/index.php/RBTCC/ article/view/267/207

Falcone, E. M. O. (2012). O papel da tomada de perspectiva na experiência da empatia. In E. M. O. Falcone, A. D. Oliva, \& C. Figueiredo (Eds.), Produções em terapia cognitivo-comportamental (pp. 61-69). São Paulo, SP: Casa do Psicólogo.

Falcone, E. M. O., Ferreira, M. C., Luz, R. C. M., Fernandes, C. S., D’Augustin, J. F., Sardinha, A., \& Pinho, V. D. (2008). Inventário de Empatia (IE): Desenvolvimento e validação de uma medida brasileira. Avaliação Psicológica, 7(3), 321-334. Recuperado em http://pepsic.bvsalud. org/pdf/avp/v7n3/v7n3a06.pdf
Falcone, E. M. O., Pinho, V. D., Ferreira, M. C., Fernandes, C. S., D'Augustin, J. F., Krieger, S., ...Pinheiro, L. C. (2013). Validade convergente do Inventário de Empatia (IE). Psico-USF, 18(2), 203-210. doi:10.1590/S141382712013000200004

Feshbach, N. D. (1997). Empathy: The formative years implications for clinical practice. In A. C. Bohart \& L. S. Greenberg (Eds.), Empathy reconsidered: New directions in Psychotherapy (pp. 33-59). Washington, DC: American Psychological Association.

Freedman, S., Enright, R. D., \& Knutson, J. (2005). A progress report on the Process Model of Forgiveness. In E. L. Worthington Jr. (Ed.). Handbook of forgiveness (pp. 393-406). New York: Routledge.

Gardner, H. (2002). As inteligências pessoais. In Estruturas da mente. A teoria das inteligências múltiplas (pp. 184-213). Porto Alegre, RS: Artmed.

Hauser, M. D. (2006). Moral minds: The nature of right and wrong. New York: Harper Perennial.

Hodgson, L. K., \& Wertheim, E. H. (2007). Does good emotion management aid forgiving? Multiple dimensions of empathy, emotion management and forgiveness of self and others. Journal of Social and Personal Relationships, 24, 931949. doi:10.1177/0265407507084191

Iacoboni, M. (2009). Imitation, empathy, and mirror neurons. Annual Review of Psychology, 60, 653-670. doi:10.1146/annurev. psych.60.110707.163604

Ickes, W. (1997). Introduction. In W. Ickes (Ed.), Empathic accuracy (pp. 1-16). New York: Guilford.

Lameira, A. P., Gawryszewski, L. G., \& Pereira, A., Jr. (2006). Neurônios-espelho. Psicologia USP, 17(4), 123-133. doi:10.1590/S010365642006000400007

McCullough, M. E., Rachal, K. C., Sandage, S. J., Worthington, E. L., Jr., Brown, S. W., \& Hight, T. L. (1998). Interpersonal forgiving in close relationships: II. Theoretical elaboration and measurement. Journal of Personality and Social Psychology, 75(6), 1586-1603. doi:10.1037/0022-3514.75.6.1586

Oliva. A. D. (2012). A mente sob uma ótica evolucionista: Bases do comportamento pró-social. In 
E. M. O. Falcone, A. D. Oliva, \& C. Figueiredo (Eds.), Produções em terapia cognitivo-comportamental (pp. 51-59). São Paulo, SP: Casa do Psicólogo.

Pfeifer, J. H., \& Dapretto, M. (2009). "Mirror, mirror, in my mind": Empathy, interpersonal competence, and the mirror neuron system. In J. Decety \& W. Ickes (Eds.), The Social Neuroscience of Empathy (pp. 183-197). Cambridge, MA: A Bradford Book.

Pinho, V. D., \& FaIcone, E. M. O. (2015). Intervenciones para la promoción del perdón y la inserción de la empatía: revisión de la literatura. Revista Argentina de Clínica Psicológica, 24(2), 111-120.

Preston, S. D., \& de Waal, F. B. M. (2002). Empathy: Its ultimate and proximate bases.Behavioral and Brain Sciences, 25, 1-72. doi 10.1017/ S0140525X02000018

Rameson, L. T., \& Lieberman, M. D. (2009). Empathy: A social cognitive neuroscience approach. Social and Personality Psichology Compass, 3, 94-110. doi:10.1111/j.1751-9004.2008.00154.x

Ribeiro, C. M., Pinho, V. D., \& Falcone, E. M. O. (2011). A influência da raiva e da empatia sobre a satisfação conjugal. Aletheia, 35-36, 7-21. Recuperado em http://www.ulbra.br/psicologia/ files/aletheia35-36.pdf
Sardinha, A., Falcone, E. M. O., \& Ferreira, M. C. (2009). As relações entre a satisfação conjugal e as habilidades sociais percebidas no cônjuge. Psicologia: Teoria e Pesquisa, 25(3), 395-402. doi:10.1590/S0102-37722009000300013

Subkoviak, M. J., Enright, R. D., Wu, C., Gassin, E. A., Freedman, S., Olson, L. M., \& Sarinopoulos, I. (1995). Measuring interpersonal forgiveness in late adolescence and middle adulthood. Journal of Adolescence, 18, 641-655. doi:10.1006/ jado.1995.1045

Worthington, E. L., Jr. (2005). Initial questions about the art and science of forgiving. In E. L. Worthington Jr. (Ed.), Handbook of forgiveness (pp. 1-13). New York: Routledge.
Recebido: 02/03/2015

$1^{a}$ revisão: 09/12/2015

Aceite final: 10/12/2015 